\title{
MACROSTYLOPHORA KINABALUAE N. SP. (INSECTA : SIPHONAPTERA : CERATOPHYLLIDAE), puce nouvelle de Sabah (Île de Bornéo, Malaisie Orientale)
}

\author{
BEAUCOURNU J.C.* \& WELLS K.**
}

Summary: MACROSTYLOPHORA KINABALUAE N. SP. (INSECTA:

Siphonaptera: Ceratophyllidae), a NEW FleA From SABAH (Borneo, EASTERN MALAYSIA)

This species, known only by a single male, is described from Mount Kinabalu and thus is recorded from the same area as Macrostylophora borneensis Uordan, 1926), teste Traub (1972). It is distinguished from its congeneric and characterized by the absence of eriged setae on the thorax and first abdominal tergits, as well as by the shape of terminal segments and the phallosom. Macrostylophora kinabaluae was found to parasitize the Sciurid rodent Callosciurus prevostii that is widespread throughout most areas in the Malaysian subregion.

KEY WORDS : Macrostylophora kinabaluae n. sp., Siphonaptera, Ceratophyllidae, Sabah, Borneo.

N ous avons récemment décrit de Sabah, et très précisément de la même région, trois Pygiopsyllidae du genre Medwayella Traub, 1972, en signalant dans nos collectes la présence d'un mâle de Macrostylophora Ewing, 1929: "Nous pouvons seulement affirmer qu'il ne s'agit pas de M. borneensis (Jordan, 1926), connu cependant du Mont Kinabalu. Le paradoxe est que ce genre compte 19 espèces décrites et une dizaine en attente de description, sans parler des sous-espèces (Traub et al., 1983). Une seule, citée ci-dessus, est nommément connue de Bornéo, alors que quatre sont notées par Durden \& Traub (1990) de cette île" (Beaucournu \& Wells, 2004).

Cet exemplaire avait été mis en attente, dans l'espoir de trouver du matériel complémentaire, ce qui ne fut pas le cas. Nous l'étudions ici, avec comme matériel de comparaison :

- un mâle, paratype de "Ceratophyllus borneensis Jordan, 1926", Mont Murud, prêté par le British Museum (Nat. Hist.);

- un mâle et une femelle de Macrostylophora borneensis (Jordan, 1926), Mont Kinabalu, donnés et

* Laboratoire de Parasitologie et Zoologie appliquée, Faculté de Médecine, 2, avenue du Professeur Léon Bernard, 35043 Rennes Cedex, France, et Institut de Parasitologie de l'Ouest, même adresse. ** Department of Experimental Ecology (Bio III), University of Ulm, Albert-Einstein Allee 11, D-89069 Ulm.

Correspondance : Pr Jean-Claude Beaucournu.

E-mail : jbeaucou@univ-rennes1.fr

\section{Résumé :}

Cette espèce, seulement connue par un mâle, est décrite du Mont Kinabalu et est donc en sympatrie avec Macrostylophora borneensis (Jordan, 1926), teste Traub (1972). Elle se caractérise par l'absence de soies érigées sur le thorax et les premiers tergites abdominaux, la forme de ses segments terminaux et le phallosome. Macrostylophora kinabaluae a été trouvé sur le rongeur Sciuridae Callosciurus prevostii, répandu dans la plus grande partie de la sous-région malaise.

MOTS CLÉS : Macrostylophora kinabaluae n. sp., Siphonaptera, Ceratophyllidae, Sabah, Bornéo.

identifiés, par notre regretté collègue, R. Traub en 1974 ;

- quatre mâles et trois femelles de M. borneensis, même origine que ci-dessus, de la collection R. Traub déposée au Carnegie Museum of Natural History à Pittsburgh (USA), confiés par son Curateur, le Professeur J.E. Rawlins;

- un mâle et une femelle de $M$. levis (Jordan et Rothschild, 1922), Pahang, Malaya, donnés et identifiés par R. Traub en 1974.

\section{MACROSTYLOPHORA KINABALUAE N. SP.}

Matériel récolté : mâle holotype, et unique, sur Callosciurus prevostii (Desmarest, 1822) (Rodentia: Sciuridae), Kinabalu Park, Poring Hot Spring, alt. ca $600 \mathrm{~m}$ (06 02.248' N-116 42.482' E), Sabah, 17XII 2002 (K.W. réc.). Ce Parc entoure le Gunong Kinabalu qui culmine à $4101 \mathrm{~m}$.

Dépôt du Type : dans les collections du premier auteur, ultérieurement déposées au Laboratoire d'Entomologie du Muséum national d'Histoire naturelle de Paris.

\section{DESCRIPTION DU MÂLE}

Capsule céphalique conforme à la redescription du genre par Jordan (1939), dans sa forme comme dans sa sétation, et dans la longueur du palpe labial.

Thorax : prothorax avec un rang de soies et une cténidie de 19 épines ; mésothorax : deux rangs de soies, dont les longues distales, et deux pseudosetae de 


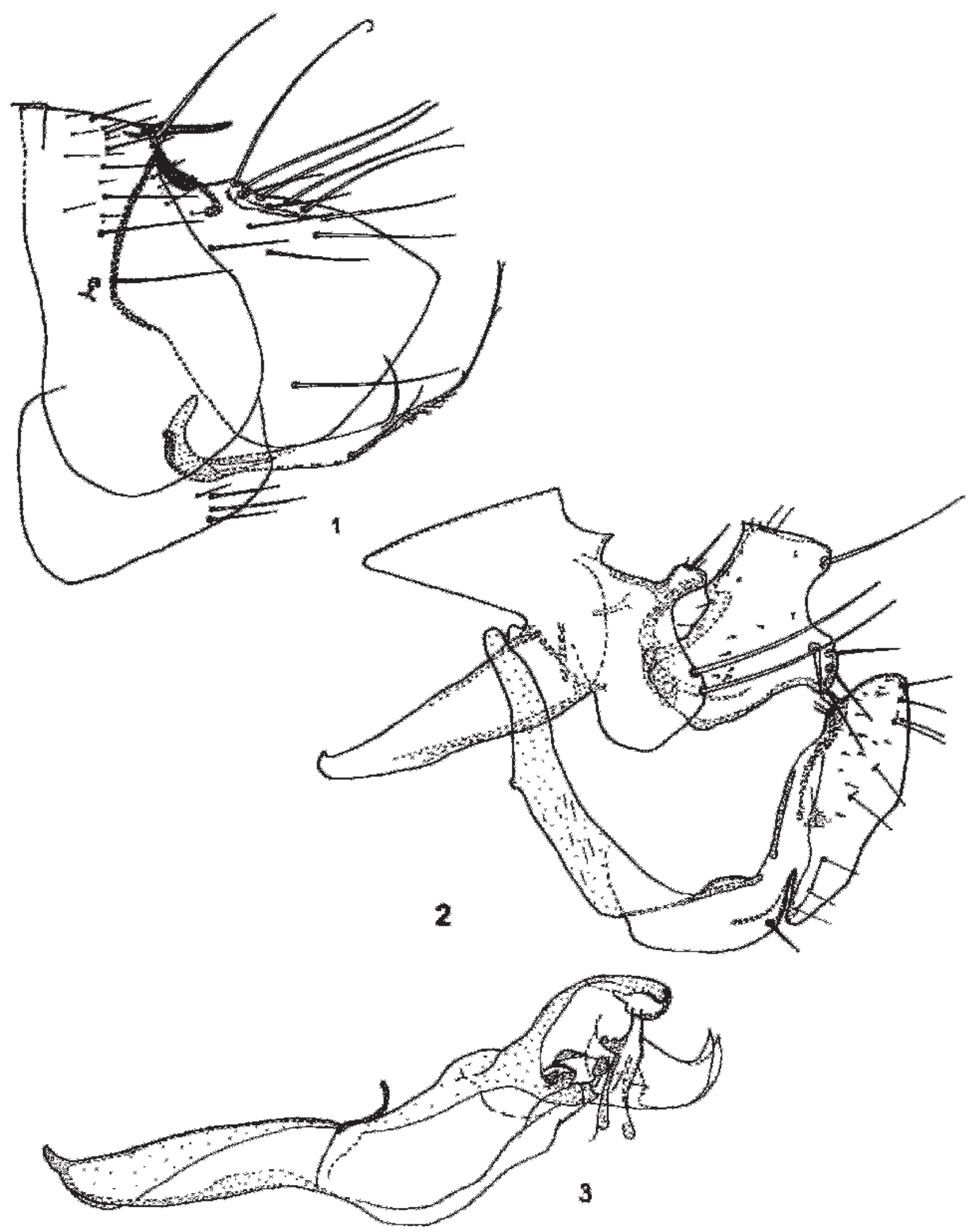


chaque côté ; métathorax : un rang de soies, plus les longues distales.

Abdomen (segments non modifiés). De même que sur les trois segments thoraciques, aucune soie n'est érigée sur les premiers segments abdominaux (elles le sont chez le mâle de $M$. borneensis). Spiracles ronds et très petits, du métépimeron au tergite VII. Deux spinules sur les tergites I à III; une seule sur les tergites IV à V. Deux rangs de soies, le distal composé de soies longues, leur apex atteignant, ou presque, l'insertion des soies longues du segment suivant; la soie inférieure est insérée au niveau du spiracle. Sétation des sternites : II, 0; III à VI, trois soies.

Abdomen (segments modifiés). Le segment VII (figure 1), ou plus précisément le tergite VII, est "stylophore" ( $c f$. Jordan, 1939); à l'inverse de M. borneensis, ce prolongement sclérifié est presque aussi long que le bord dorsal du tergite; une seule soie antésensiliale sur un petit socle, flanquée de deux courtes et fortes soies. Sternite portant dans son angle inféro-distal trois soies développées et, une, supérieure, plus petite. Segment VIII (figure 1) : le tergite est grand et porte sur sa marge dorsale six soies longues et érigées, plus six autres plus courtes dans ce quadrant supérieur. Une aire spiculeuse interne, de présence classique, s'étend le long de la marge dorsale, en dessous des longues soies; elle est seulement formée de deux lignes sub-parallèles (chez M. borneensis, c'est une véritable plage). Une seule soie, longue, dans la partie basale mais non marginale du tergite. Les deux tiers postérieurs du bord dorsal sont très finement crénelés. Sternite grêle : il porte quelques micro-soies et une soie longue à son apex, lequel se termine par un long et fin vexillum, peu ramifié. La glande de Wagner est présente et est très grande; son plus grand diamètre est égal à la moitié de la hauteur du basimère; cette glande manque chez M. borneensis. Tergite IX (figure 2) : manubrium à base large, sa longueur dépassant l'apex de l'apodème; on note à sa base un petit tubercule triangulaire. Le basimère est trapu, comme chez les autres espèces de ce genre, et porte deux longues soies acétabulaires. Le télomère apparaît dans son ensemble plus ou moins rectangulaire, mais avec une forte protubérance en "bec de perroquet" vers le milieu de son bord postérieur; cette protubérance porte une courte et forte soie externe et trois internes et marginales; une "protubérance" de même aspect général peut exister chez $M$. borneensis, mais elle est située à l'angle inféro-distal. À l'angle supéro-distal, on note une soie interne, marginale, longue. Le sternite IX a un bras proximal classique; la partie distale ne se caractérise que par sa largeur (environ deux fois plus large que le bras proximal). Phallosome (figure 3) : lamina media longue, à bords sub-parallèles; lobe dorso-médian en volute; crochets développés, de courbe arrondie vers le haut, à apex très acuminés.
Femelle inconnue.

Dimension (insecte monté) : 2,5 mm.

Discussion

Si l'on ne veut considérer que les espèces de Macrostylophora décrites de l'île de Bornéo, la validité de M. kinabaluae est incontournable, du moins chez le mâle, seul sexe à notre disposition : soies du thorax et des premiers tergites non érigées; espèce "stylophore"; présence d'une glande de Wagner; différences morphologiques entre les segments IX de borneensis et de kinabaluae. La distinction, par rapport aux Macrostylophora d'autres régions, est cependant à examiner pour le principe.

Macrostylophora levis (Jordan et Rothschild, 1922) semble, à première vue, très proche, sinon identique. Cette puce n'est connue, pour autant que nous le sachions, que de Malaisie péninsulaire (Selangor, terra typica; Pahang). Cependant, le segment IX est, inter alia, bien différent : mâle non stylophore; soie antésensiliale plus longue; processus basimeris dorsalis beaucoup plus long; sternite plus étroit; crochets du phallosome acuminés, certes, mais étroits et allongés. À notre avis, les autres taxa décrits ne méritent pas de discussion. La description de "Ceratophyllus borneensis" par Jordan montre, chez l'unique femelle à sa disposition, un sternite VII "deeply sinuate the upper lobe narrow and projecting much more than the ventral lobe". Les femelles attribuées à $M$. borneensis par Traub (il n'y a pas de publication spécifique à ce sujet) ont une marge postérieure du sternite VII ne montrant qu'un très petit lobe, surmontant une concavité largement ouverte.

Il faut souligner enfin, pour en terminer avec cette discussion, que toutes les Puces citées de cette région, Macrostylophora comme Medwayella, sont inféodées aux Sciuridae, ou secondairement, en particulier dans nos récoltes, aux Tupaiidae (Scandentia). Toutes ces espèces font partie d'une cohorte de petits mammifères très divers montrant des chevauchements importants dans l'occupation de l'habitat, autant dans "l'axe" vertical qu'horizontal (Wells et al., 2004).

Notons, à propos des "écureuils", que Li \& Traub (1998) ont décrit le sous-genre Songshupsylla ("songshu" signifiant écureuil en chinois), pour les Macrostylophora Ewing, 1929 ne possédant pas, chez le mâle, de prolongement net entre les soies antésensiliales; ces auteurs y ajoutent divers autres critères, mais reconnaissent, comme l'avait déjà écrit Jordan (1939), qu'aucun caractère n'est suffisamment constant chez les femelles pour permettre, en l'absence de mâle, leur attribution à ce sous-genre ou au sous-genre nominatif. Nous adhèrerons donc à la position prudente de ce dernier auteur qui, pour rester cohérent avec les règles de nomenclature, conserva "Macrostylophora", mais estima qu'il s'agissait d'un nom générique mal choisi car, entre autres, l'ornementation "stylophore" des mâles ne 
concerne qu'une partie des taxa connus. On peut noter que pour ce genre malchanceux, Songshupsylla est aussi mal venu que Macrostylophora, puisque, si le genre entier est primitivement inféodé aux écureuils, d'autres hôtes "de capture" existent, dont, nous l'avons dit, les Tupaiidae.

Pour ce qui concerne l'hôte de $M$. kinabaluae, le Sciuridae Callosciurus prevostii, sa répartition couvre "à l'exception de Java, toute la sous-région malaise y inclus le nord du Sulawesi (Célèbes), bien que cette dernière population ait, peut-être, été introduite" (Hoffmann et al., 1993).

\section{REMERCIEMENTS}

Toute notre gratitude va à ceux de nos collègues qui ont bien voulu, soit nous donner des exemplaires de leur collection, en l'occurrence le regretté Pr. R. Traub, soit nous confier pour études comparatives des exemplaires figurant dans les Collections dont ils sont curateurs: Miss Th. Howard (London, UK) et le Professeur J.E. Rawlins (Pittsburgh, USA).

Par ailleurs, nous remercions l'Economic planning unit Malaysia pour nous avoir accordé un Permis de Recherche à Sabah. Nous sommes également reconnaissants aux Sabah Parks, comme au Yayasan Sabah pour leurs autorisations de travailler dans plusieurs zones forestières et pour leurs diverses aides bienveillantes sur le terrain. Le support financier, pendant le séjour de l'un de nous (K.W.) à Sabah, fut aimablement apporté par le Deutscher Akademischer Austauschdienst (DAAD).

\section{RÉFÉRENCES}

Beaucournu J.C. \& Wells K. Trois espèces nouvelles du genre Medwayella Traub, 1972 (Insecta : Siphonaptera : Pygiopsyllidae) de Sabah (Malaisie Orientale, Île de Bornéo), Parasite, 2004, 11, 373-377.

Durden L.A. \& Traub R. Zoogeographical implications from rodent ectoparasites in Sulawesi (pp. 51-62). In: Insects and the rain forests of South East Asia, Wallacea. Knight W.J. \& Holloway J.D. (eds.), The Royal Entomological Society of London, 1990, 4, 343 p.

Hoffmann R.S., Anderson C.G., Thorington R.W. JR. \& Heaney L.R. Family Sciuridae (pp. 419-465). In: Mammal species of the World, a taxonomic and geographic reference, $2^{\text {th }}$ ed. Wilson D.E. \& Reeder D.A.M. (eds), Smithsonian Institution Press, Washington, London, 1993, XVI, 1207 p.

JordAn K. New Siphonaptera. Novitates Zoologicae, 1926, 33, 385-394.

JORDAN K. Siphonaptera collected by Dr. Gerd Heinrich in Burma. Novitates Zoologicae, 1939, 41, 362-375.

Jordan K. \& Rothschild N.C. New species of Siphonaptera collected by Mr. C. Boden-Kloss in the Malay peninsula ans south Annam. Ectoparasites, 1922, 1, 217-230.
Li Kuen-Chen \& Traub R. Description of Songshupsylla, a new subgenus of Macrostylophora (Siphonaptera: Ceratophyllidae), with a discussion of its species groups. Journal of Medical Entomology, 1998, 35, 396-398.

Traub R. The Gunong Benom Expedition, 1967: 11. Notes on zoogeography, convergent evolution and taxonomy of Fleas (Siphonaptera), based on collections from Gunong Benom and elsewhere in South-East Asia. I. New taxa (Pygiopsyllidae, Pygiopsyllinae). Bulletin of the British Museum (Natural History), Zoology, 1972, 23, 203-305 + 58 pl.

Wells K., Pfeiffer M., Lakim M.B. \& Linsenmair K.E. Use of arboreal and terrestrial space by a small mammal community in a tropical rain forest in Borneo, Malaysia. Journal of Biogeography, 2004, 31, 641-652.

Reçu le 17 mars 2005 Accepté le 22 avril 2005 